\title{
Personalizing tumour vaccines
}

Immunotherapies targeting cytotoxic T-lymphocyte-associated antigen-4 (CTLA-4) and programmed death-1 (PD-1) or its ligand PD-L1 have shown encouraging efficacy. However, our knowledge of the tumour-specific mutant antigens (TSMAs) that drive these responses remains poor. Now, two studies have described similar methodologies for identifying tumour antigens.

In both studies, potential MHC I-binding TSMA peptides were identified in different mouse tumour models by combining exome sequencing, mass spectrometry, and in silico predictive modelling data; in some tumour cell lines, thousands of potential TSMAs were refined to a single-digit number of epitopes likely to have the capacity to evoke an antitumour immune response. These promising TSMAs were validated as $\mathrm{CD}^{+} \mathrm{T}$-cell targets in vivo by assessing $\mathrm{T}$-cell responses after immunization with synthetic TSMA peptides and by staining tumour-infiltrating lymphocytes (TILs) with MHC I-TSMA peptide complexes, identifying 0-3 key TSMAs in each model.

"We asked whether these TSMAs were the targets of the T cells unleashed by treatment with the immune checkpoint blocking antiCTLA-4 or anti-PD-1 antibodies," explains Robert Schreiber, who led one of the studies. The data support this hypothesis; in particular, the key functional TSMAs were detected by mass spectrometry in the mouse sarcoma model used, and staining with the MHC ITSMA peptide complexes revealed that TIL populations increased over time after anti-PD-1 therapy, reaching highest levels just before tumour rejection.

Importantly, both studies found that the key TSMA peptides could drive considerable antitumour immune responses, with synthetic peptide vaccines containing the relevant epitopes resulting in tumour rejection in mice.

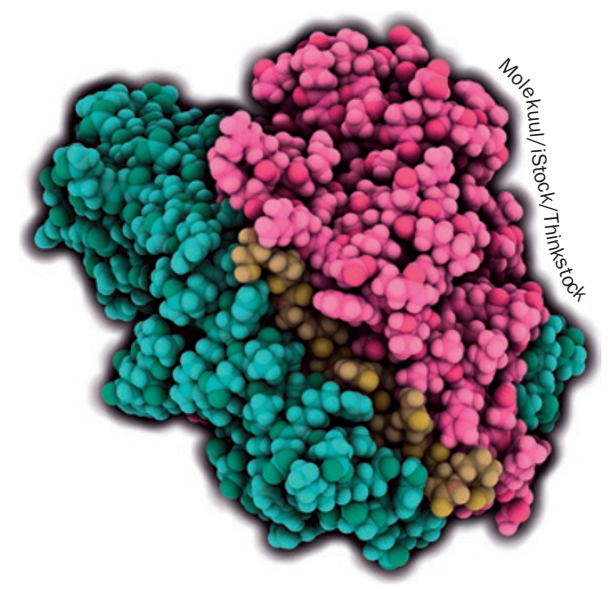

"Therapeutic vaccines comprising multiple epitopes derived from different TSMAs can be as effective as checkpoint blockade therapy," states Schreiber. "This would make an interesting strategy therapeutically, allowing one to target mutated peptides specific to the tumour, leaving nonmalignant cells unscathed," adds Jennie Lill, who co-led the other study with Lélia Delamarre.

Furthermore, these findings have implications regarding biomarkers for immunotherapy. As Schreiber explains, "being able to identify $T$ cells with specificity for relevant TSMAs, we can study the changes that occur in the T-cell population following different types of immunotherapy and get a relatively accurate picture of whether the therapy will be successful (that is, the changes that occur in tumour-specific T cells are the best biomarkers of response to therapy)."

"We are now planning to validate our approach in human cancers and would also like to develop an approach that is entirely computational to predict immunogenic TSMAs," concludes Delamarre.

David Killock

Original articles Yadav, M. et al. Predicting immunogenic tumour mutations by combining mass spectrometry and exome sequencing. Nature 515, 572-576 (2014) | Gubin, M. M. et al. Checkpoint blockade cancer immunotherapy targets tumour-specific mutant antigens. Nature 515, 577-581 (2014) 